\title{
Simultaneous Multimode Oscillation of Stress-Compensated Cut Quartz Resonator with Narrow-Band Wide Variable-Range Quartz Crystal Oscillator
}

\author{
Tomio Sato, Tetsuya Akitsu \\ Division of Human Environmental and Medical Engineering, Interdisciplinary Graduate School of Medicine and \\ Engineering, University of Yamanashi, Kofu, Japan \\ Email: akitsu@yamanashi.ac.jp
}

Received 16 September 2014; revised 12 October 2014; accepted 5 November 2014

Academic Editor: Yongdong Li, Department of Mechanical Engineering, Academy of Armored Force Engineering, China

Copyright (C) 2014 by authors and Scientific Research Publishing Inc.

This work is licensed under the Creative Commons Attribution International License (CC BY). http://creativecommons.org/licenses/by/4.0/

(c) (i) Open Access

\begin{abstract}
A multimode-quartz-crystal oscillator was developed to excite stable dual-mode resonance at different frequencies: The oscillation of the 3rd harmonic resonance of the principle C-mode and an additional resonance B-mode of SC-cut crystal. Harmonic combinations of the 3rd and fundamental mode of B-mode with the 3rd harmonics of $\mathrm{C}$-mode are demonstrated. The measurement of the temperature dependence of the oscillation frequency is demonstrated along with the stability determined by root Allan variance. Dependence on the open conductance of the active circuit and the dependence on the coupling capacitors are discussed.
\end{abstract}

\section{Keywords}

Temperature Sensing, Piezoelectric Sensor, Dual-Mode Quartz Crystal Resonance, Stress-Compensated Cut (SC-Cut)

\section{Introduction}

Piezoelectric sensing is increasingly attracting attentions, employing frequency-based measurement depending on the surface loading, mass, and a variety of force acting on the piezoelectric resonator. The quartz crystal resonator determines a stable electrical resonance frequency corresponding to the mechanical resonance frequency. 
The quartz crystal resonator shows a variety of resonance modes. For example, in an AT-cut quartz crystal, the harmonic combination of the different temperature characteristics of the fundamental, 3rd and 5th harmonic resonances provides a high precision thermometric system. In the past history, dual-mode oscillation was attempted by several groups and is reviewed in References [1]-[6]. In Reference [3], a thermometry method is presented, using a harmonically related pair of C-modes of an SC-cut quartz crystal resonator. SC cut is produced with a double rotated cut angle. One of the benefits of SC cut application is stress compensation, which can provide a solution for the temperature shock sensitivity and multimode oscillation: providing primary mode oscillation with high stability called C-mode and additional resonance B-mode located at 9\% higher frequency. The experiment is realized by the combination of oscillation circuit with passive frequency discrimination circuits or heterodyne method observing a mixed signal response of the quartz crystal resonator. Simultaneous multimode observation in the crystal provides larger information, because this mode acts as an embedded thermometer having a frequency shift proportional to temperature, which in turn enables precise control of the temperature stabilizing the oscillation frequency and precise control of the oscillation frequency and the stability. Recently, we described the development of a narrow-band quartz crystal oscillator circuit [7]. C-mode is aimed as a stable frequency standard and B-mode is aimed as a thermometry method. The dual-mode quartz crystal oscillator in the combination of the 3rd harmonics oscillations of C-mode and the 3rd harmonics of B-mode needs the discrimination of narrower frequency separation, compared with the combination of the fundamental oscillation of B-mode. In this work, stable dual-mode quartz crystal oscillator circuit was developed based on a combination of two identical narrow gain quartz crystal oscillators. Measurement of frequency stability is based on the Allan deviation for discrete multiple measure determined following Barnes et al. [8] and Allan [9]. The Allan deviation in the simultaneous dual-mode oscillation and the oscillation condition for multiple oscillation modes are presented following IEEE Standard 1139 [10].

\section{Dual Mode Quartz Crystal Oscillator}

\subsection{Analysis of Dual Mode Quartz Crystal Oscillator}

Figure 1 shows the dual-mode oscillation circuit. Individual oscillator circuits are developed from a narrowband, double-resonance quartz crystal oscillator [7].

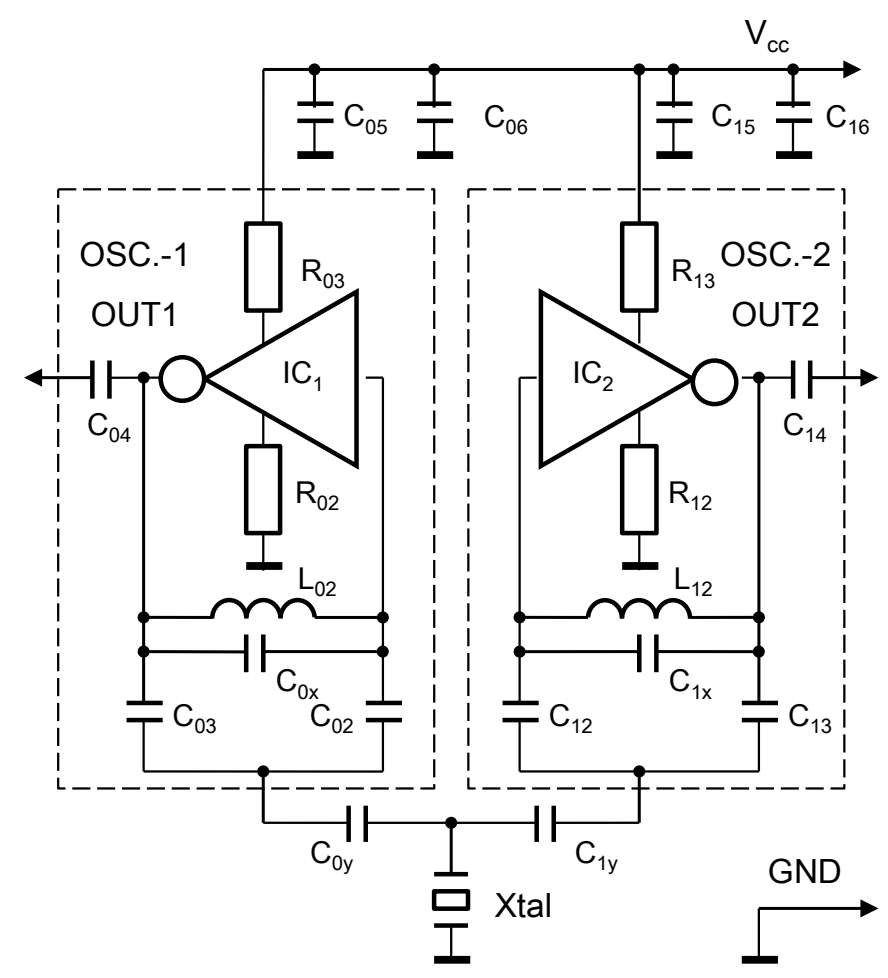

Figure 1. Dual mode quartz crystal oscillator. 
Local resonance circuits consist of $L_{02}$ and $C_{0 x}, L_{12}$ and $C_{1 x}$. $C_{0 y}$ and $C_{1 y}$ are inserted to the connection to a crystal resonator. Feedback resistors $R_{02}, R_{03}, R_{12}$, and $R_{13}$ are inserted into the power line between CMOS inverter integrated circuits $\mathrm{IC}_{1}$ and $\mathrm{IC}_{2}$ and ground aimed at suppression of current and gain control. $C_{05}, C_{06}, C_{15}$, and $C_{16}$ are pass capacitors. $C_{02}, C_{03}, C_{12}$, and $C_{13}$ are necessary for the generation of negative resistance. This circuit synthesizes LC oscillation mode determined by $L_{02}$ and combined capacitors $C_{0 x}, C_{02}, C_{03}$, and $C_{0 y}$, and LC oscillation mode determined by $L_{12}$ and combined capacitors $C_{1 x}, C_{12}, C_{13}$, and $C_{1 y}$. The oscillation frequency is settled in the vicinity of the quartz crystal oscillation frequency. Coupling capacitors $C_{04}$ and $C_{14}$ are omitted in the following analysis. Coupling capacitors $C_{0 y}$ and $C_{1 y}$ are connected to the quartz crystal resonator. Appropriate choice of the local resonance circuit and the coupling capacitors are necessary to realize stable dual-mode quartz crystal oscillation. Two oscillator circuits $\mathrm{OSC}_{1}$ and $\mathrm{OSC}_{2}$ are connected to the resonator by capacitors $\mathrm{C}_{0 y}$ and $\mathrm{C}_{1 y}$. The SC-cut quartz crystal resonator shows C-mode a primary oscillation mode with good stability, and B-mode, a side mode, linearly proportional to the change of ambient temperature. CMOS Inverters $\mathrm{IC}_{1}$ and $\mathrm{IC}_{2}$ are replaced with a constant current source. In the equivalent circuit on the left side is $\mathrm{OSC}_{1}$ and on the right side is $\mathrm{OSC}_{2} . I_{\text {out1 }}$ and $I_{\text {out2 }}$ are output current, and $V_{\mathrm{in} 1}$ and $V_{\mathrm{in} 2}$ are input voltage of each inverter: $r_{02}$ and $\mathrm{r}_{12}$ are internal resistance of inductance $L_{02}$ and $L_{12}$, respectively. $C_{0 s}$ and $C_{1 s}$ are stray capacitance parallel to capacitance $C_{0 x}$ and $C_{1 s}$. Impedance $Z_{01}$ consists of $L_{02}, r_{02}, C_{0 x}$, and $C_{0 s}$, similarly, impedance of the circuit $Z_{11}$ which consists of $L_{12}, r_{12}, C_{1 x}$, and $C_{1 s}$, and $Z_{x t}$ for the parallel circuit of a quartz resonator and $C_{p}$, reduced impedance is found. Capacitance of leads and connected lines on the circuit board are modeled by $C_{p}$. Applying Kirchhoff's law, homogeneous Equation (1) is found.

$$
\left[\begin{array}{ccccccc}
1 & 0 & 0 & -1 & 0 & 0 & -1 \\
0 & 1 & -1 & 1 & 0 & 0 & 0 \\
0 & Z_{01}+Z_{02} & Z_{03} & 0 & 0 & 0 & 0 \\
G_{1 M} Z_{x t} & -G_{1 M} Z_{02} & 0 & 1+G_{1 M} Z_{0 y} & 0 & 0 & 0 \\
0 & 0 & 0 & 0 & 1 & -1 & 1 \\
0 & 0 & 0 & 0 & Z_{11}+Z_{12} & Z_{13} & 0 \\
G_{2 M} Z_{x t} & 0 & 0 & 0 & -G_{2 M} Z_{12} & 0 & 1+G_{2 M} Z_{1 y}
\end{array}\right]\left[\begin{array}{c}
i_{x t} \\
i_{01} \\
i_{03} \\
i_{0 y} \\
i_{11} \\
i_{13} \\
i_{1 y}
\end{array}\right]=\left[\begin{array}{l}
0 \\
0 \\
0 \\
0 \\
0 \\
0 \\
0
\end{array}\right]
$$

The open conductance $G_{m}$ is adjusted by negative feedback resistors $R_{02}, R_{03}, R_{12}$, and $R_{13}$. Equivalent transconductance is defined by the open conductance and a feedback resistance, as in (2).

$$
G_{1 M}=\frac{I_{\text {out1 }}}{V_{\text {in } 1}}, G_{2 M}=\frac{I_{\text {out2 }}}{V_{\text {in2 }}} .
$$

The open conductance of the CMOS inverter is expressed with drain current $I_{d}$ and conductance coefficient $K$ defined in the terms of electron and hole mobility $\mu$ and the unit gap capacitance $C_{g}$. W/L is the ratio of width over length of the gate:

$$
G_{i M}=\frac{G_{m}}{1+G_{m} R_{i f}}, G_{m}=2 g_{m}, g_{m}=2 \sqrt{K I_{d}}, \text { where } K=\frac{1}{2} \mu C_{g} \frac{W}{L}
$$

Feedback resistance:

$$
R_{1 f}=R_{02}=R_{03}, R_{2 f}=R_{12}=R_{13}
$$

Solving the determinant of the coefficient matrix, relation (3) is found. The second term on the left side of this relation indicates the impedance of $\mathrm{OSC}_{1}$, and the third term indicates the impedance of $\mathrm{OSC}_{2}$. The impedance of individual oscillator is connected in parallel to $Z_{x t}$.

$$
\frac{1}{Z_{x t}}+\frac{1}{\frac{1}{G_{1 M}}+\frac{Z_{02} Z_{03}}{Z_{01}+Z_{02}+Z_{03}}+Z_{0 y}}+\frac{1}{\frac{1}{G_{2 M}}+\frac{Z_{12} Z_{13}}{Z_{11}+Z_{12}+Z_{13}}+Z_{1 y}}=0
$$

Figure 2 shows the entire structure of the oscillator equivalent circuit-2, indicating the impedance of $\mathrm{OSC}_{1}$ as $Z_{1}$ and the impedance of $\mathrm{OSC}_{2}$ as $Z_{2}, R_{1}, R_{2}, C_{1}$, and $C_{2}$ are equivalent resistance and capacitance of the imped- 


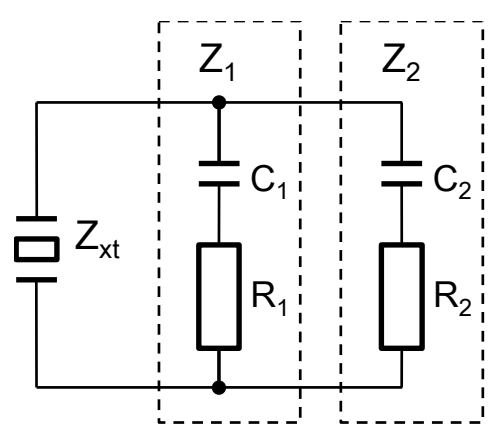

Figure 2. Equivalent circuit-2.

ance. $R_{1}, C_{1}, R_{a}, L_{a}, C_{0 x s}, \omega_{0 x}, \omega_{0 s}$, and $\omega_{1}$ are found in (4). $R_{2}, C_{2}, R_{b}, L_{b}, C_{1 x s}, \omega_{1 x}, \omega_{1 s}$, and $\omega_{2}$ are found in (5).

$$
\begin{aligned}
& Z_{1}=\frac{1}{G_{1 M}}+\frac{Z_{02} Z_{03}}{Z_{01}+Z_{02}+Z_{03}}+Z_{0 y}=R_{1}+\frac{1}{j \omega C_{1}}, Z_{2}=\frac{1}{G_{2 M}}+\frac{Z_{12} Z_{13}}{Z_{11}+Z_{12}+Z_{13}}+Z_{1 y}=R_{2}+\frac{1}{j \omega C_{2}} . \\
& R_{1}=\frac{1}{G_{1 M}}-\frac{1}{\omega^{2} C_{02} C_{03}} \frac{R_{a}}{R_{a}^{2}+\left\{\omega \cdot L_{a}\left(1-\frac{\omega_{1}^{2}}{\omega^{2}}\right)\right\}^{2}}, \frac{1}{C_{1}}=\frac{1}{C_{0 y}}-\frac{1}{C_{02} C_{03}} \frac{L_{a}\left(1-\frac{\omega_{1}^{2}}{\omega^{2}}\right)}{R_{a}^{2}+\left\{\omega \cdot L_{01}\left(1-\frac{\omega_{1}^{2}}{\omega^{2}}\right)\right\}^{2}} \\
& R_{a}=\frac{r_{02}}{\left(1-\frac{\omega^{2}}{\omega_{0 x}^{2}}\right)^{2}+\left(\frac{\omega}{\omega_{0 s}}\right)^{2}}, L_{a}=L_{02} \frac{1-\frac{\omega^{2}}{\omega_{0 x}^{2}}-\frac{\omega_{0 x}^{2}}{\omega_{0 s}^{2}}}{\left(1-\frac{\omega^{2}}{\omega_{0 x}^{2}}\right)^{2}+\left(\frac{\omega}{\omega_{0 s}}\right)^{2}} . \\
& C_{0 x s}=C_{0 x}+C_{0 s} \text {, } \\
& \omega_{0 x}^{2}=\frac{1}{L_{02} C_{0 x s}}, \omega_{0 s}=\frac{1}{r_{02} C_{0 x s}}, \omega_{1}^{2}=\frac{1}{L_{a}}\left(\frac{1}{C_{02}}+\frac{1}{C_{03}}\right) .
\end{aligned}
$$

Similarly,

$$
R_{2}=\frac{1}{G_{2 M}}-\frac{1}{\omega^{2} C_{12} C_{13}} \frac{R_{b}}{R_{b}^{2}+\left\{\omega L_{b}\left(1-\frac{\omega_{2}^{2}}{\omega^{2}}\right)\right\}^{2}}, \frac{1}{C_{2}}=\frac{1}{C_{1 y}}-\frac{1}{C_{12} C_{13}} \frac{L_{b}\left(1-\frac{\omega_{2}^{2}}{\omega^{2}}\right)}{R_{b}^{2}+\left\{\omega \cdot L_{b}\left(1-\frac{\omega_{2}^{2}}{\omega^{2}}\right)\right\}^{2}}
$$

where

$$
\begin{gathered}
R_{b}=\frac{r_{12}}{\left(1-\frac{\omega^{2}}{\omega_{1 x}^{2}}\right)^{2}+\left(\frac{\omega}{\omega_{1 s}}\right)^{2}}, L_{b}=L_{12} \frac{1-\frac{\omega^{2}}{\omega_{1 x}^{2}}-\frac{\omega_{1 x}^{2}}{\omega_{1 s}^{2}}}{\left(1-\frac{\omega^{2}}{\omega_{1 x}^{2}}\right)^{2}+\left(\frac{\omega}{\omega_{1 s}}\right)^{2}} . \\
C_{1 x s}=C_{1 x}+C_{1 s}, \\
\omega_{1 x}^{2}=\frac{1}{L_{12} C_{1 x s}}, \omega_{1 s}=\frac{1}{r_{12} C_{1 x s}}, \omega_{2}^{2}=\frac{1}{L_{b}}\left(\frac{1}{C_{12}}+\frac{1}{C_{13}}\right) .
\end{gathered}
$$

Including the equivalent circuit of $Z_{x t}$, equivalent circuit-3 is found in Figure 3, where $L_{1}, C_{1}$, and $R_{1}$ are the motion arm and $C_{0}$ is the parallel capacitance. $C_{p}$ is stray capacitance on the circuit board connected to the resonator. Solving for $Z_{x t}$, equivalent resistance $R_{c i}$ and equivalent reactance $C_{c i}$ of parallel connection of $Z_{1}$ and $Z_{2}$ 


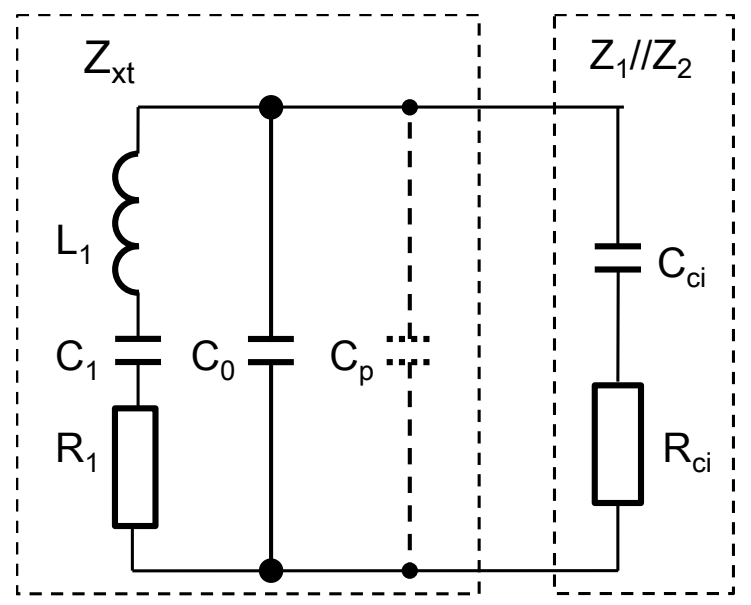

Figure 3. Equivalent circuit-3.

are found.

$$
Z_{x t}+\frac{Z_{1} Z_{2}}{Z_{1}+Z_{2}}=Z_{x t}+R_{c i}+\frac{1}{j \omega C_{c i}}=0
$$

where

$$
R_{c i}=\frac{R_{1} R_{2}\left(R_{1}+R_{2}\right)+\frac{1}{\omega^{2}}\left(\frac{R_{2}}{C_{1}^{2}}+\frac{R_{1}}{C_{2}^{2}}\right)}{\left(R_{1}+R_{2}\right)^{2}+\frac{1}{\omega^{2}}\left(\frac{1}{C_{1}}+\frac{1}{C_{2}}\right)^{2}}, \frac{1}{C_{c i}}=\frac{\frac{R_{2}^{2}}{C_{1}}+\frac{R_{1}^{2}}{C_{2}}+\frac{1}{\omega^{2} C_{1} C_{2}}\left(\frac{1}{C_{1}}+\frac{1}{C_{2}}\right)}{\left(R_{1}+R_{2}\right)^{2}+\frac{1}{\omega^{2}}\left(\frac{1}{C_{1}}+\frac{1}{C_{2}}\right)^{2}} .
$$

Figure 4 shows a simplified graphical expression of equivalent circuit-4, where capacitance $C_{0 p}$ consists of $C_{0}$ and $C_{p}$, and $C_{c c i}$ consists of $C_{c i}$ and $C_{z}$. The whole circuit consists of elements $C_{0}, L_{1}, C_{1}$, and $R_{1}$ representing the equivalent circuit of the selected mode of the SC-cut quartz crystal resonator (values are in Table 1).

Including $C_{0}, C_{p}$ and $C_{z}$ into $C_{c i}$ and $R_{c i}$ composed equivalent impedance $C_{c c i}$ and $R_{c c i}$ are found, as in (7).

$$
\begin{aligned}
& R_{c c i}=\frac{R_{c i}}{\left(1+\frac{C_{0 p}}{C_{c i}}\right)^{2}+\left(\omega \cdot C_{0 p} R_{c i}\right)^{2}}, \frac{1}{C_{c c i}}=\frac{1}{C_{0 p}}\left\{1-\frac{1+\frac{C_{0 p}}{C_{c i}}}{\left(1+\frac{C_{0 p}}{C_{c i}}\right)^{2}+\left(\omega \cdot C_{0 p} R_{c i}\right)^{2}}\right\} . \\
& C_{0 p}=C_{0}+C_{p} .
\end{aligned}
$$

Supposing $i(\omega)$ in the closed circuit of this diagram, the resistance condition is found from the matching condition for the real part where the real part is equal to zero. Oscillation frequency is determined from the matching condition of the capacitance, where the imaginary part is equal to zero.

\subsection{Oscillation Conditions: Combination of Fundamental Resonance and the 3rd Harmonics}

Oscillation conditions are given as the resistance condition and frequency condition, in the following forms (8) and (9), respectively:

$$
\begin{gathered}
R_{1}+R_{c c i} \leq 0, \quad R_{1} \leq\left|R_{c c i}\right| \\
\omega^{2}=\frac{1}{L_{1}}\left(\frac{1}{C_{1}}+\frac{1}{C_{c c i}}\right)
\end{gathered}
$$




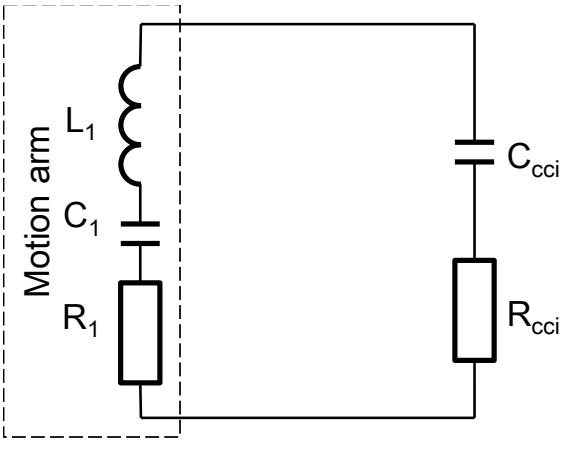

Figure 4. Equivalent circuit-4.

Table 1. Equivalent circuit constant of the SC cut quartz crystal resonator.

\begin{tabular}{ccccccc}
\hline \multirow{2}{*}{ Mode } & \multicolumn{5}{c}{ Equivalent Circuit Constant } \\
\cline { 2 - 7 } & $C_{0}(\mathrm{pF})$ & $L_{1}(\mathrm{mH})$ & $C_{1}(\mathrm{fF})$ & $R_{1}(\Omega)$ & $f_{1}(\mathrm{MHz})$ & $f_{r}(\mathrm{MHz})$ \\
\hline C-mode, 3rd harmonics & 3.431 & 1297 & 0.1954 & 70.5 & 9.997418 & 9.999807 \\
B-mode 3rd harmonics & 3.244 & 1757 & 0.12 & 264 & 10.96083 & 10.952481 \\
B-mode fundamental & 3.899 & 495.3 & 3.710 & 61.8 & 3.712778 & 3.712836 \\
\hline
\end{tabular}

Inequality in condition (8) indicates that the negative resistance larger than damping is necessary at the beginning of oscillation. The series resonance frequency $f_{1}$ is determined by $L_{1}$ and $C_{1}$ determined in relation (10), where $\omega_{1}$ is angular frequency and $f_{r}$ is the series resonance frequency.

$$
\omega_{1}^{2}=\frac{1}{L_{1} C_{1}}, \omega_{1}=2 \pi f_{1} .
$$

The resistance condition is estimated for the oscillation of each mode from Table 1, the value of series resistance $R_{1 c}$ and $R_{1 b}$ determined by the impedance analyzer. Relation (11) shows resistance conditions for the 3rd harmonic resonance modes. The frequency condition shows the resonance frequency determined by $L_{1 c}$ and $C_{1 c}$ or $L_{1 b}$ and $C_{1 b}$. $C_{1 c}$ and $C_{1 b}$ are extremely smaller than $C_{c c i}$.

$$
R_{1 c}=70.5 \Omega \leq\left|R_{c c i}\right|, R_{1 b}=264 \Omega \leq\left|R_{c c i}\right|
$$

In the case of the fundamental resonance of B-mode, the series resistance is reduced to $61.77 \Omega$. Negative resistance of the active circuit is indicated by the absolute value. In the physical meaning, this value balances with the damping; this value is expressed in the following form:

$$
\left|R_{c c i}\right|,\left(R_{c c i}<0\right)
$$

Figure 5 shows the tuning characteristics by changing resonator capacitors $C_{0 x}$ and $C_{1 x}$ combined with $L_{02}$ and $L_{12}$, which determine the oscillation frequency of the individual oscillation mode.

The frequency dependence of negative resistance is tailored considering the influence of resonator capacitors $C_{0 x}$ and $C_{1 x}$. The oscillation condition of the 3rd harmonics of C-mode is fulfilled at $C_{1 x}=20.5 \mathrm{pF}$, where the oscillation frequency is close to the series resonance frequency. The maximum absolute value of negative resistance is approximately $1.99 \mathrm{k} \Omega$, at $9.992 \mathrm{MHz}$. The frequency range varies from 0.28 to $0.3 \mathrm{MHz}$. Similarly, the oscillation condition of the 3rd harmonics B-mode is fulfilled at $C_{0 x}=13.5 \mathrm{pF}$, close to the series resonance frequency $f_{1 b}$. The maximum absolute value of negative resistance is approximately $1.9 \mathrm{k} \Omega$, at $10.9 \mathrm{MHz}$. The oscillation condition is fulfilled from 10.84 to $10.96 \mathrm{MHz}$. The frequency range varies from 0.11 to $0.12 \mathrm{MHz}$. In the combination of the fundamental oscillation of B-mode and the 3rd harmonic resonance of C-mode, the dual mode oscillation is realized at $C_{0 x}=31 \mathrm{pF}$ and $C_{1 x}=20.5 \mathrm{pF}$.

In the combination of the 3rd harmonic resonance modes, the multimode-oscillation is observed provided that the oscillation condition is fulfilled simultaneously. In the stable oscillation, gain $G_{1 M}$ decreases compared with 


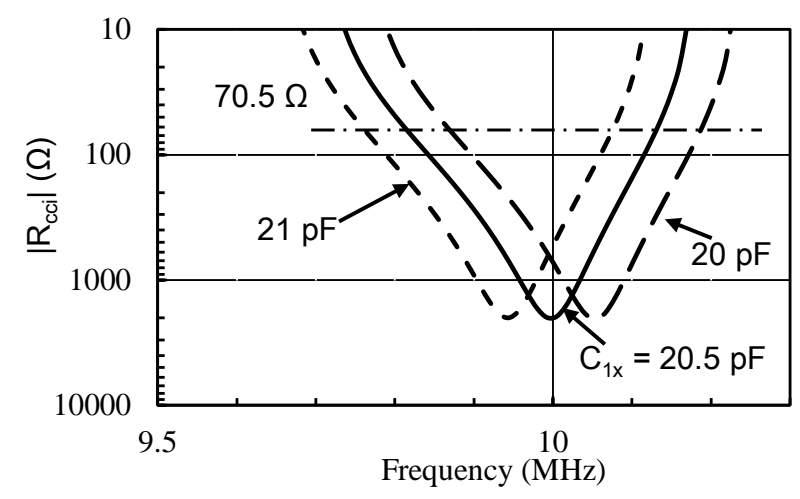

Figure 5. Frequency dependence of negative resistance for local resonator capacitors: $C_{0 x}$ and $C_{1 x}$.

the initial phase. At $G_{1 M}=1 \mathrm{~mA} / \mathrm{V}$, the damping resistance becomes larger than negative resistance. The maximum absolute value of negative resistance is approximately equal to $2 \mathrm{k} \Omega$ and the damping resistance of the 3rd harmonic resonance of B-mode is $264 \Omega$. The negative resistance of the oscillator is shown for various values of gain $G_{1 M}$, in the vicinity of $10.9 \mathrm{MHz}$ and $10 \mathrm{MHz}$.

In Figure 6(a) and Figure 6(b), as the gain decreases in the stable oscillation at $G_{1 M}=1 \mathrm{~mA} / \mathrm{V}$, the negative resistance becomes smaller than $R_{1 b}$ then the oscillation condition is no longer fulfilled, while the oscillation condition for C-mode still holds. Transition of oscillation mode occurs from dual-mode oscillation to the single oscillation. Figure 6(c) and Figure 6(d) shows the oscillation condition the gain of C-mode oscillator $G_{2 M}$. $G_{2 M}$ is settled to $5 \mathrm{~mA} / \mathrm{V}$ initially, and the gain of the counterpart oscillator B-mode oscillator $G_{1 M}$ is settled to 5 $\mathrm{mA} / \mathrm{V}$. The maximum absolute value of negative resistance is approximately $2 \mathrm{k} \Omega$. Generally, the transconductance $G_{M}$ of the oscillator circuit decreases in the magnitude on the growth of signals, and the frequency dependence of negative resistance varies depending on $G_{M}$. In the case where $G_{1 M}$ of $\mathrm{OSC}_{1}$ is equal to $0 \mathrm{~mA} / \mathrm{V}$, negative resistance of $\mathrm{OSC}_{2}$ shows wide-frequency characteristics and decreases in the frequency according to the increase in the magnitude of $G_{1 M}$. Negative resistance of $\mathrm{OSC}_{1}$ shows an abrupt change in the vicinity of $G_{1 M}=1$ $\mathrm{mA} / \mathrm{V}$. Negative resistance of $\mathrm{OSC}_{2}$ shows corresponding change but the oscillation condition is fulfilled. Similar tendency is observed in the dependence of negative resistance of $\mathrm{OSC}_{1}$ according to the decrease in conductance $G_{2 M}$ of $\mathrm{OSC}_{2}$.

The gain at the initial stage of oscillation is set at $5 \mathrm{~mA} / \mathrm{V}$, along with the growth of the signal, B-mode shows a maximum value of negative resistance $4.5 \mathrm{k} \Omega$ at $G_{1 M}$ of approximately $2 \mathrm{~mA} / \mathrm{V}$, then steeply decreases. In Figure 7(a), negative resistance shows zero at $G_{1 M}$ approximately $1.8 \mathrm{~mA} / \mathrm{V}$. As the negative resistance of Cmode is larger than $1 \mathrm{k} \Omega$, a maximum value $3.4 \mathrm{k} \Omega$ is observed at $G_{1 M}$ approximately $0.5 \mathrm{~mA} / \mathrm{V}$, the oscillation condition is fulfilled for this mode, and the oscillation mode transfers to C-mode and B-mode becomes incapable. Similarly in Figure 7(b), the gain settled to $5 \mathrm{~mA} / \mathrm{V}$ at the initial stage of oscillation, and the negative resistance shows zero at $G_{2 M}$ approximately $1.0 \mathrm{~mA} / \mathrm{V}$. As the negative resistance of B-mode is larger than $0.7 \mathrm{k} \Omega$, a maximum $4.7 \mathrm{k} \Omega$ at $G_{1 M}$ of $1.3 \mathrm{~mA} / \mathrm{V}$, the oscillation mode transfers to the single mode oscillation of B-mode when the condition of $\mathrm{C}$-mode becomes unfulfilled. When the 3rd harmonic resonance of B-mode is selected, the resistance is $264 \Omega$, and the oscillation region becomes narrower, because larger negative resistance is needed due to the dependence on $G_{1 M}$. Because the dependence on $G_{2 M}$ is fulfilled, the oscillation region is limited by the the B-mode oscillator. Figure 7(c) and Figure 7(d) show the case of the combination of B-mode fundamental versus the 3rd harmonics of C-mode. In this case, the series resistance of B-mode decreases to $61.77 \Omega$.

Figure 8 shows the dependence on the transconductance for the case two parameters are varied simultaneously. Initially, gain $G_{1 M}$ and $G_{2 M}$ are settled to $5 \mathrm{~mA} / \mathrm{V}$. When the gain $G_{1 M}$ decreases to $2.6 \mathrm{~mA} / \mathrm{V}$, negative resistance for B-mode shows $4.8 \mathrm{k} \Omega$. Then it shows a maximum value $0 \Omega$ at $G_{1 M}=2.1 \mathrm{~mA} / \mathrm{V}$. Similarly, C-mode shows $2.9 \mathrm{k} \Omega$, at $G_{2 M}=1.8 \mathrm{~mA} / \mathrm{V}$. Then, it shows a maximum $0 \Omega$ at $G_{1 M}=1.15 \mathrm{~mA} / \mathrm{V}$. The maximum value of negative resistance is located at a certain value of gain, which is not necessarily large. In another view of the oscillation conditions, quartz crystal oscillation is stabilized at minimum negative resistance. The minimum value of negative resistance $\left|R_{c c i}\right| \min$ is $264 \Omega$ for B-mode and $70.5 \Omega$ for C-mode, from Table 1 . At $\left|R_{c c}\right|$ min larger than 

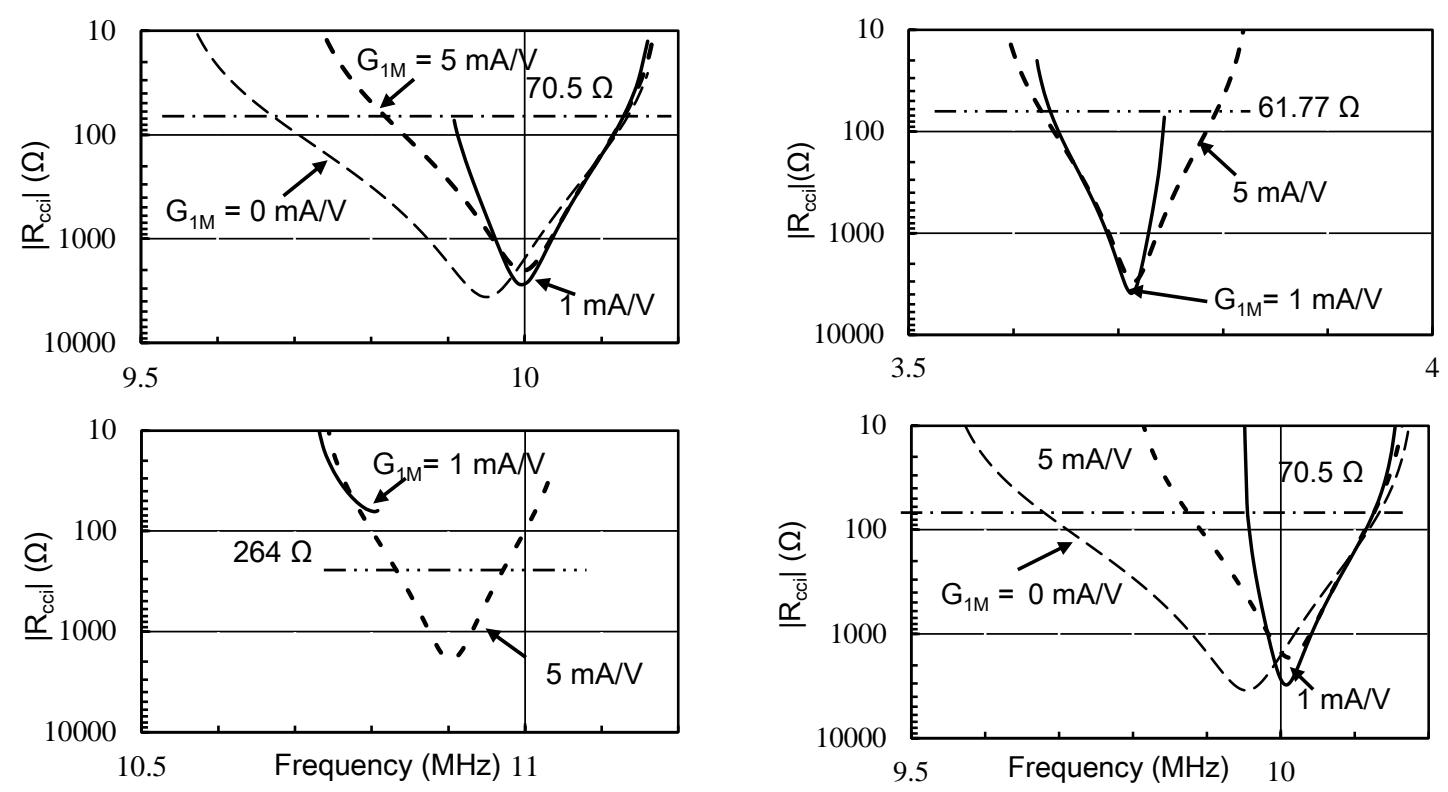

(a)
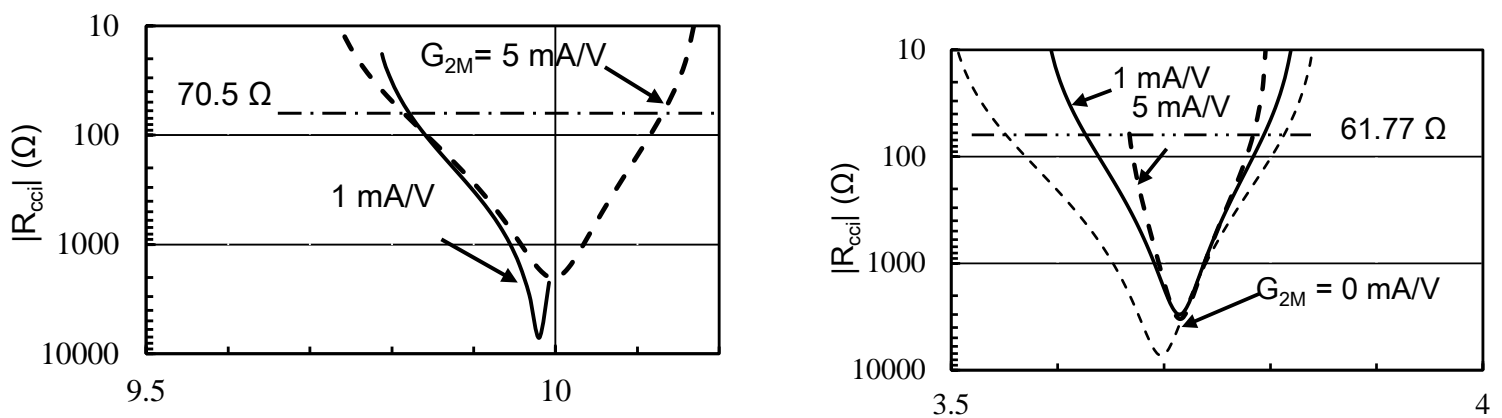

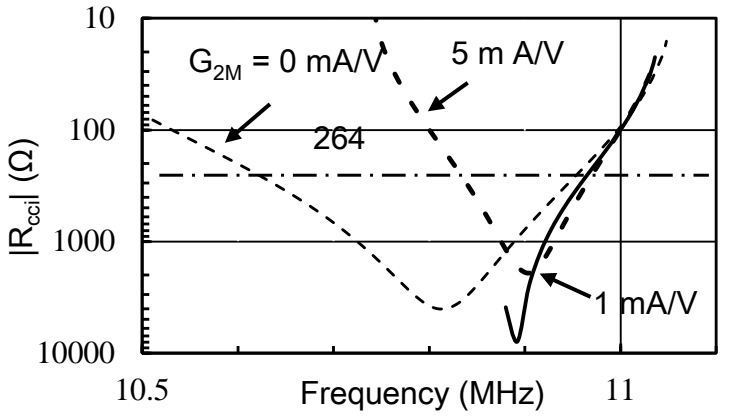

(c)

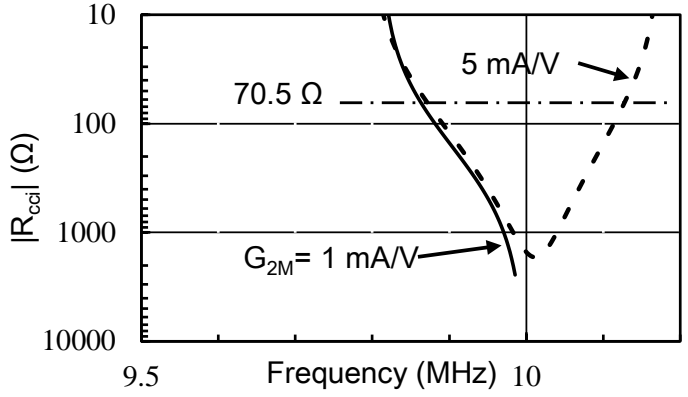

(d)

Figure 6. Depencence of negative resistance on transconductance: $G_{1 M}$ and $G_{2 M}$. (a) Negative resistance in the combination of the 3rd harmonics B-mode vs. 3rd C-mode; (b) Negative resistance in the combination of B-mode fundamental vs. the 3rd harmonics C-mode; (c) Negative resistance in the combination of the 3rd harmonics B-mode vs. 3rd C-mode; (d) Negative resistance in the combination of B-mode fundamental vs. the 3rd harmonics C-mode.

$70.5 \Omega$ the oscillation condition of C-mode is fulfilled before the oscillation condition of B-mode is achieved, if negative resistance is smaller than $264 \Omega$. In this case, the oscillation mode transfers to the single oscillation of C-mode.

Figure 9 shows the dependence of negative resistance on frequency for various values of coupling capacitors: $C_{0 y}$ and $C_{1 y}$.

In Figure 9(a), the case of the combination of the 3rd harmonic resonance of B-mode and C-mode, $C_{0 y}$ is 


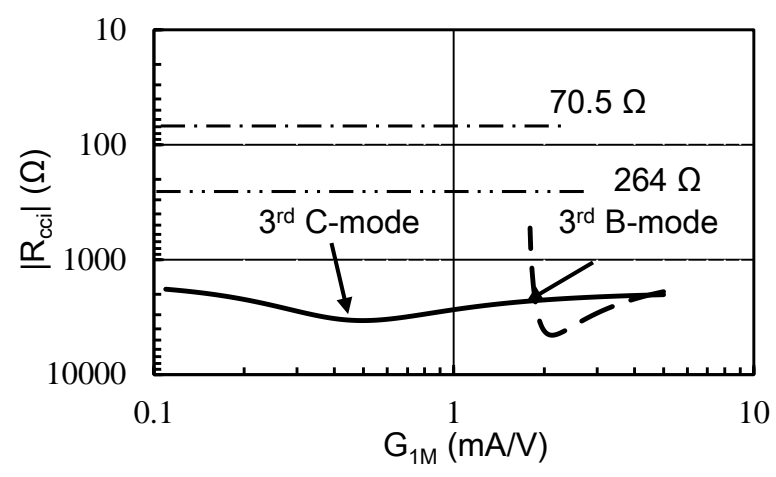

(a)

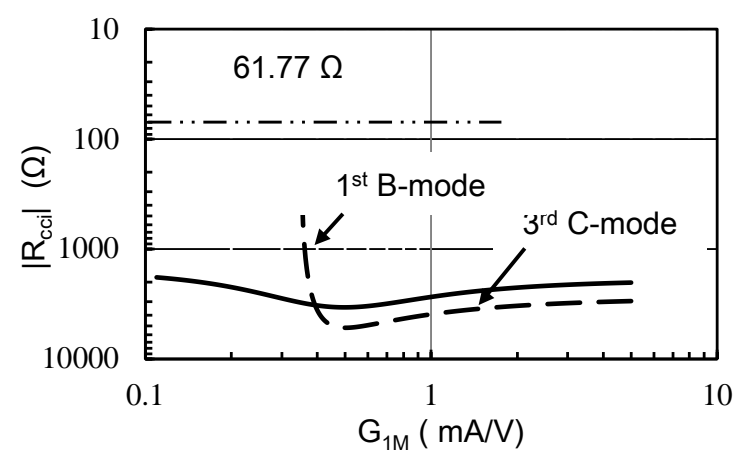

(c)

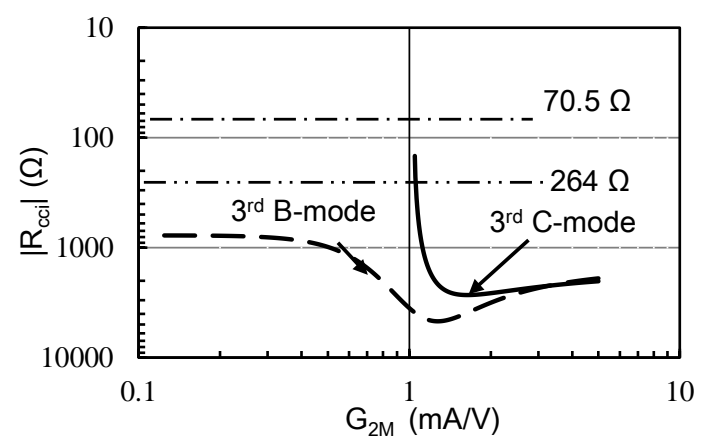

(b)

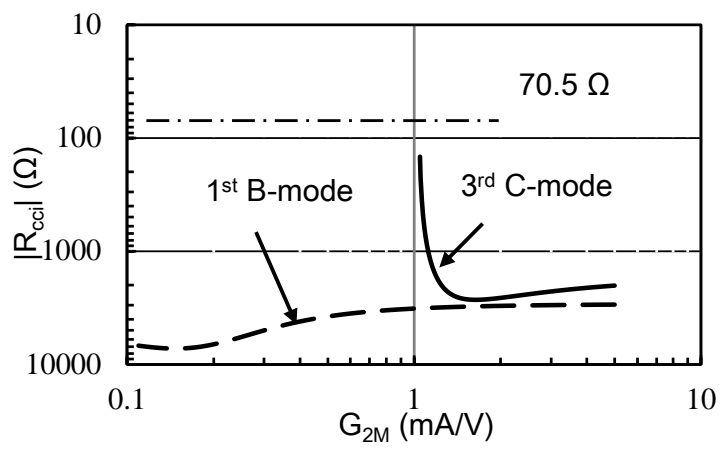

(d)

Figure 7. Dependence of negative resistance on gain: $G_{1 M}$ and $G_{2 M}$. (a) Dependence on $G_{1 M}, G_{2 M}=5 \mathrm{~mA} / \mathrm{V}$; (b) Dependence on $G_{2 M}, G_{1 M}=5 \mathrm{~mA} / \mathrm{V}$; (c) Dependence on $G_{1 M}, G_{2 M}=5 \mathrm{~mA} / \mathrm{V}$; (d) Dependence on $G_{2 M}, G_{1 M}=5 \mathrm{~mA} / \mathrm{V}$.

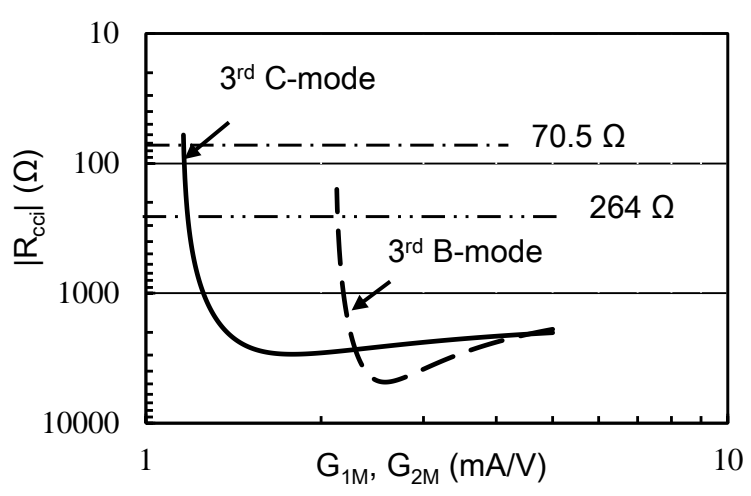

(a)

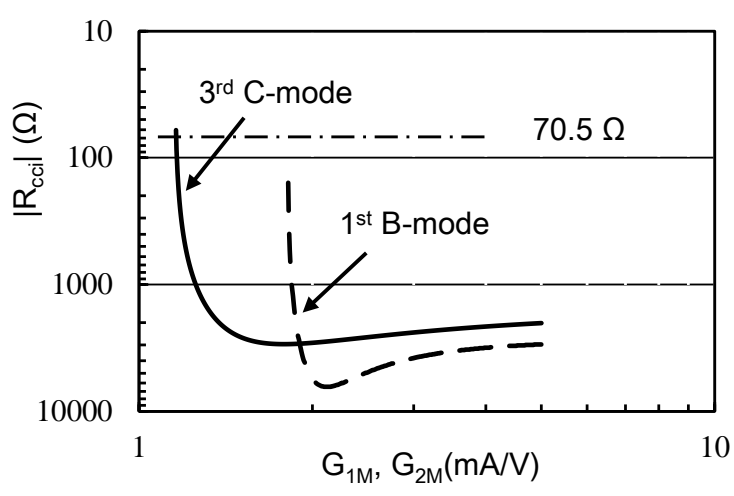

(b)

Figure 8. Dependence of negative resistance on gain: $G_{1 M}$ and $G_{2 M}$. (a) Combination of the 3rd harmonics of B-mode versus 3rd harmonics of C-mode; (b) Combination of the fundamental of B-mode versus 3rd harmonics of C-mode.

fixed at $10 \mathrm{pF}$. The case of $C_{0 y}=0 \mathrm{pF}$ means that the oscillator is disconnected from the quartz resonator and only $\mathrm{OSC}_{1}$ is connected to the resonator. The oscillation condition for B-mode $\left(\mathrm{OSC}_{1}\right)$ is fulfilled in a narrow region in the vicinity of the resonance frequency, in the case where $C_{1 y}=30 \mathrm{pF}$ :

$$
\left|R_{c c i}\right|>R_{1 b}(=264 \Omega)
$$

In Figure 9(b), the case of the combination of the fundamental B-mode versus the 3rd harmonics of C-mode,

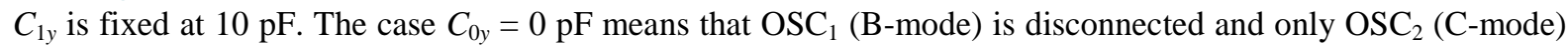
is connected with the quartz resonator. The oscillation condition for C-mode is fulfilled from 9.7 to $10.1 \mathrm{MHz}$. For $C_{0 y}=10 \mathrm{pF}$, the negative resistance of $\mathrm{OSC}_{1}$ and $\mathrm{OSC}_{2}$ is approximately equal to $2 \mathrm{k} \Omega$. The oscillation con- 

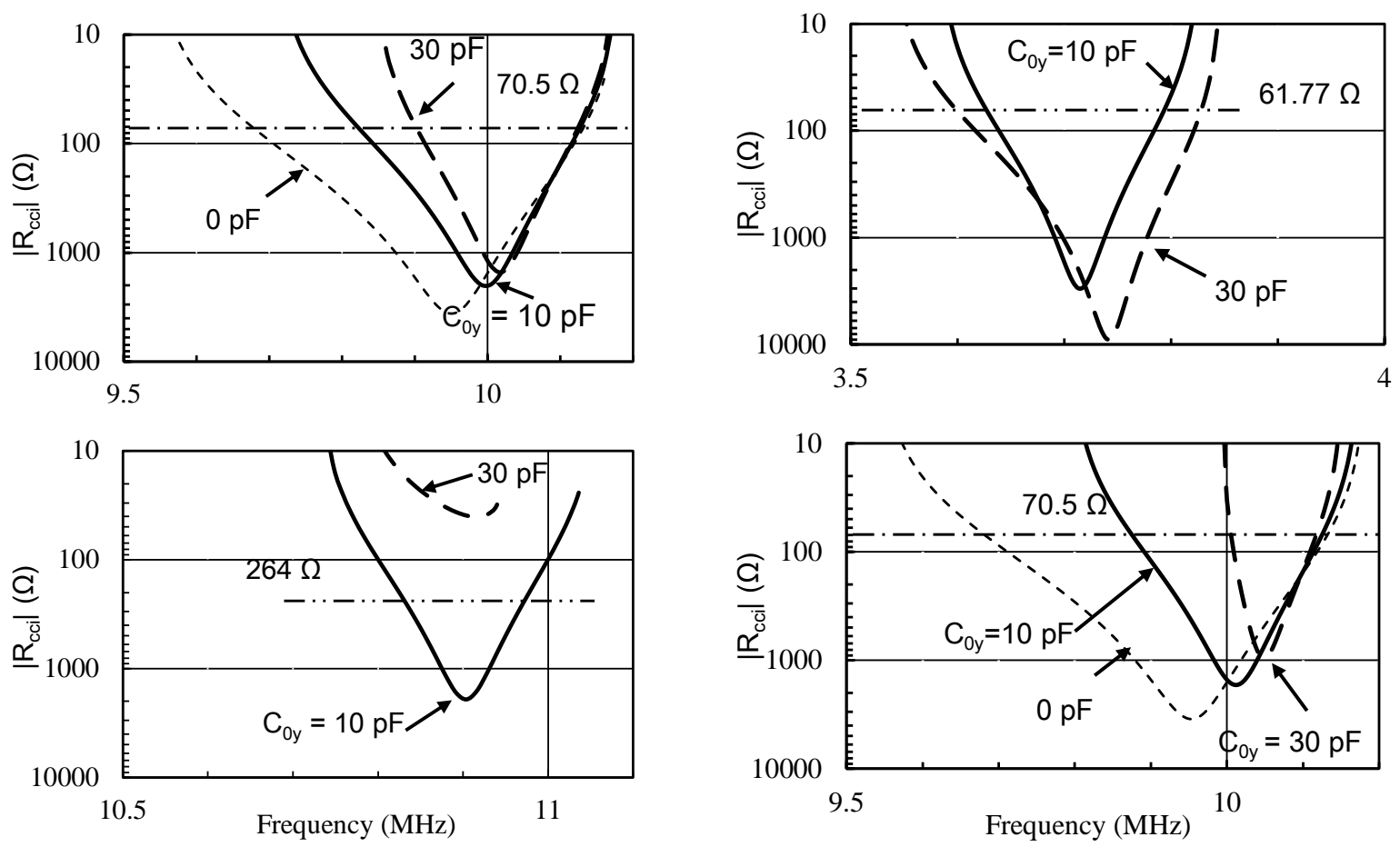

(a)

(b)
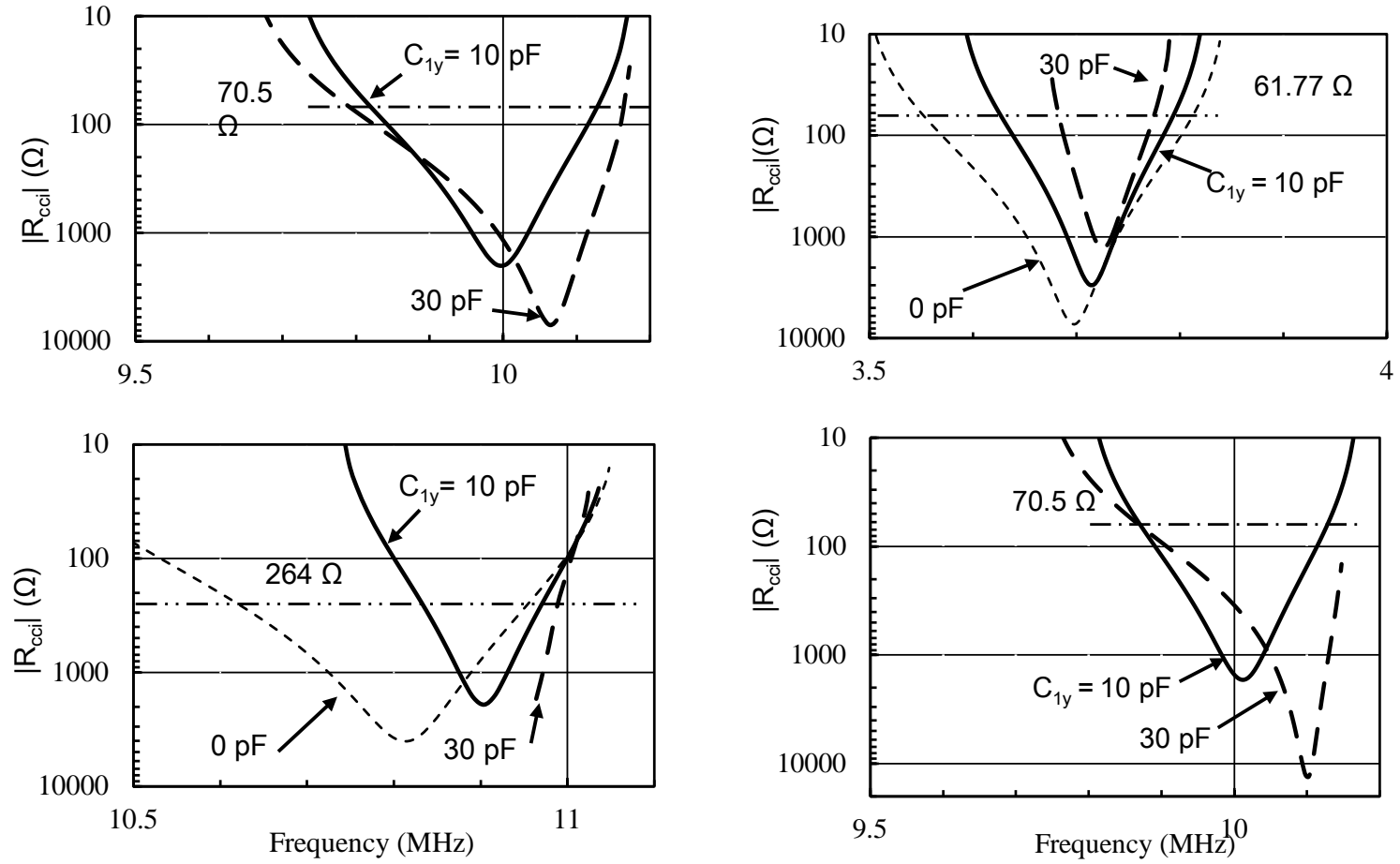

(c)

(d)

Figure 9. Dependence of negative resistance on coupling capacitors: $C_{0 y}$ and $C_{1 y}$. (a) Combination of the 3rd harmonics of B-mode versus the 3rd harmonics of C-mode. $C_{1 y}$ is fixed at $10 \mathrm{pF}$; (b) Combination of B-mode fundamental versus the 3rd harmonics of C-mode. $G_{1 M}=5 \mathrm{~mA} / \mathrm{V}$ and $G_{2 M}=5 \mathrm{~mA} / \mathrm{V} ; C_{z}=\infty \mathrm{pF}$; (c) Combination of the 3rd harmonics of B-mode versus the 3rd harmonics of C-mode. $C_{0 x}=13.5 \mathrm{pF} ; C_{0 s}=5 \mathrm{pF} ; C_{0 y}=10 \mathrm{pF}$; (d) Combination of fundamental B-mode versus the 3rd harmonics of C-mode. $C_{0 x}=31 \mathrm{pF} ; C_{1 x}=20.5 \mathrm{pF} ; C_{1 s}=5 \mathrm{pF} ; L_{12}=5.6 \mu \mathrm{H} ; G_{2 M}=5 \mathrm{~mA} / \mathrm{V} ; C_{p}=2 \mathrm{pF} ; C_{0 c}=3.431$ $\mathrm{pF} ; C_{0 b}=3.244 \mathrm{pF}$. 
dition for C-mode is fulfilled from 9.8 to $10.1 \mathrm{MHz}$ and for B-mode from 10.8 to $10.9 \mathrm{MHz}$. If $C_{0 y}$ is set to 30 $\mathrm{pF}$, the oscillation condition for $\mathrm{OSC}_{2}$ is fulfilled, but the negative resistance for $\mathrm{OSC}_{1}$ is lower than the critical value. Figure 9(c) and Figure 9(d) show the oscillation condition for $\mathrm{OSC}_{2} . C_{1 y}$ is varied while the counterpart $C_{0 y}$ is fixed at $10 \mathrm{pF}$. The peak for the $\mathrm{OSC}_{2}$ steps out of the resonance frequency region at $C_{1 y}$ equal to or larger than $30 \mathrm{pF}$.

Figure 10 shows negative resistance as functions of coupling capacitors $C_{0 y}$ and $C_{1 y}$, where $C_{0 y}$ either $C_{1 y}$ is variable and counterpart is fixed at $10 \mathrm{pF}$. $C_{1 y}=10 \mathrm{pF}$, the oscillation condition of B-mode is fulfilled from 3.56 to $3.8 \mathrm{MHz}$, and the oscillation condition of C-mode is fulfilled from 9.8 to $10.1 \mathrm{MHz}$. The relation between negative resistance $\left|R_{c c i}\right|$ and coupling capacitor $C_{0 y}$ shows that negative resistance of C-mode oscillator fulfills the oscillation condition for wide range of $C_{0 y}$.

$$
\left|R_{c c i}\right|>R_{1 c}(=70.5 \Omega)
$$

On the other hand, negative resistance of the B-mode oscillator fulfills the oscillation condition only in a narrower region from 6 to $18 \mathrm{pF}$.

$$
\left|R_{c c i}\right|>R_{1 b}(=264 \Omega)
$$

The oscillation condition of C-mode is fulfilled, at $C_{0 y}$ and $C_{1 y}$ from 2 to $48 \mathrm{pF}$, and stable oscillation of $\mathrm{C}$-mode is available. The oscillation condition of B-mode is fulfilled only at narrower region of $C_{0 y}$ and $C_{1 y}$ from 6 to $13 \mathrm{pF}$. Stable dual mode resonance oscillation is available in this region. Figure 10(b) shows the case of the combination of fundamental B-mode and the 3rd harmonics of C-mode. The oscillation condition is fulfilled in wider choice of the parameter $C_{0 y}$ and $C_{1 y}$. The value of connecting capacitors $C_{0 y}$ and $C_{1 y}$ is a parameter that enables simultaneous dual mode resonance oscillation. In summary, the best coupling capacitances are $C_{0 y}=10$ $\mathrm{pF}$ and $C_{1 y}=10 \mathrm{pF}$. If $C_{0 y}$ is larger than $30 \mathrm{pF}$, the oscillation condition for $\mathrm{OSC}_{1}$ is not fulfilled, while the oscillation condition for $\mathrm{OSC}_{2}$ is fulfilled from 9.8 to $10.2 \mathrm{MHz}$. This discussion on the choice of coupling capacitors must be reviewed in the case of the lower limit of the transconductance.
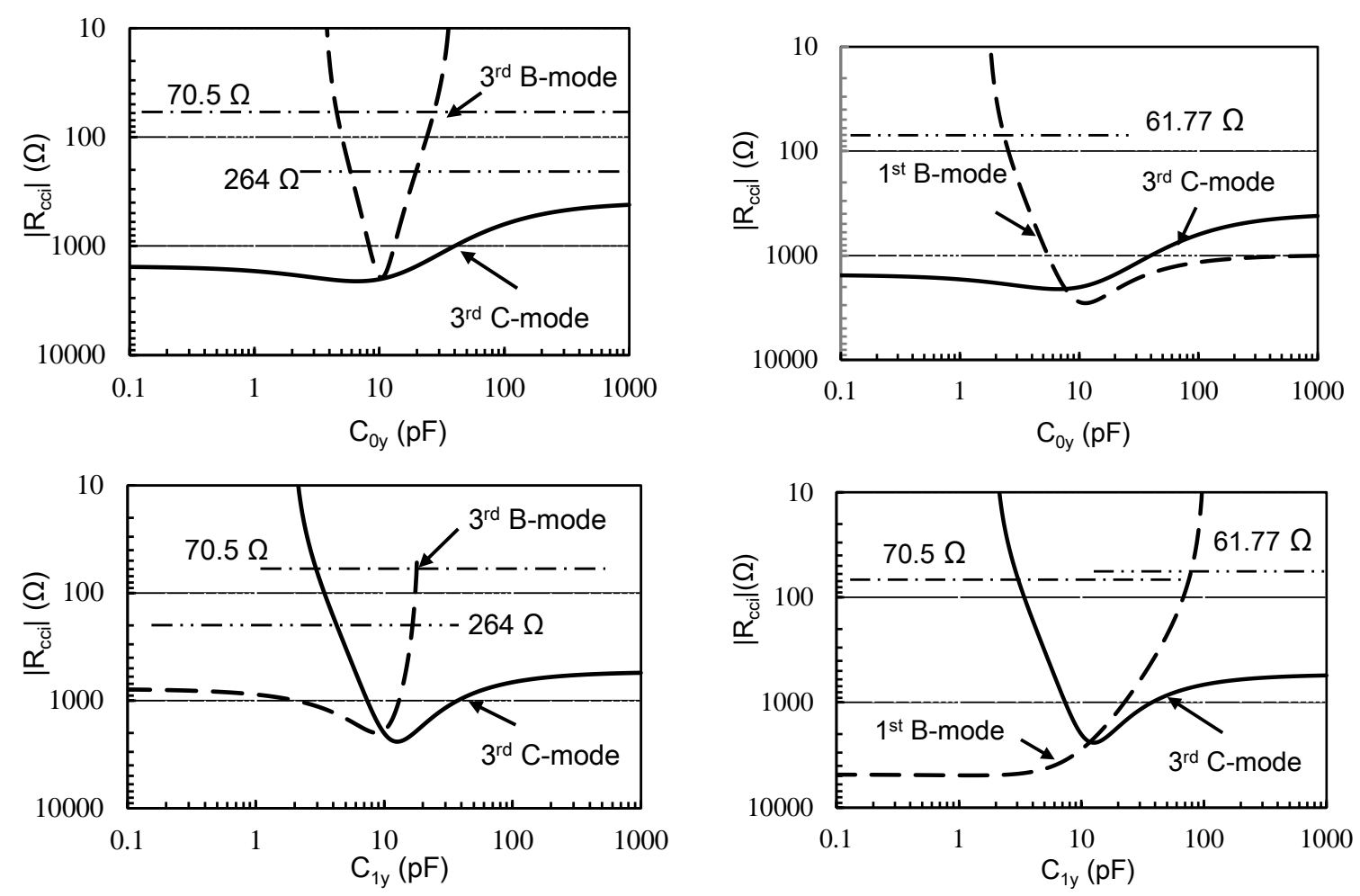

(a)

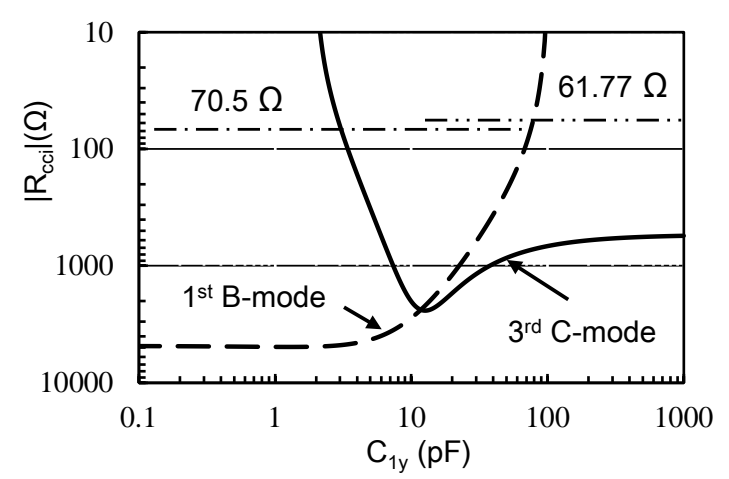

(b)

Figure 10. Dependence of negative resistance on coupling capacitors: $C_{0 y}$ and $C_{1}$. (a) Combination of the 3rd harmonics of C-mode and B-mode; (b) Combination of the fundamental B-mode and the 3rd harmonics of C-mode. 


\section{Experimental Result and Discussions}

\subsection{Analysis of the Stability}

The short-time stability of the C-mode oscillation is compared. The bottom level of the $\sigma$ versus $\tau$ dependence is employed as the measure of the stability, where $\sigma$ is the root Allan variance defined as (12) and $\tau$ is the sampling interval (gate time) [10]

$$
\sigma_{y}(\tau)=\left[\frac{2}{M-1} \sum_{k=1}^{M-1}\left(\frac{f_{k+1}-f_{k}}{f_{k+1}+f_{k}}\right)^{2}\right]^{\frac{1}{2}} .
$$

$f_{k}$ is the moving average of 10 sequential of frequency data. $\tau$ is the sampling time interval. $M$ is the number of samples per measurement. The average of frequency is calculated over the gate time. Measurement was carried out by universal frequency counters Agilent 53230A (Agilent Technologies, Santa Clara, Ca, USA) synchronized with a rubidium oscillator. Figure 11 shows typical example of wave forms observed in the simultaneous multimode oscillation.

The stability of the single C-mode oscillation is compared with the simultaneous multimode oscillation of different harmonic combinations. In this experiment, the measure of the stability is the bottom level of the $\sigma$ versus $\tau$ dependence, where $\sigma$ is the root Allan variance defined as (12) and $\tau$ is the sampling interval (gate time). Figure 12 shows the $\sigma$ versus $\tau$ curve for the oscillation of C-mode, single-mode oscillation.

Figure 13(a) shows the root Allan variance of the 3rd harmonics of C-mode $\sigma_{y}(\tau)<10^{-10}$ at $\tau$ of $100-1000$ ms and the 3rd harmonics of B-mode $\sigma_{y}(\tau)<10^{-10}$ for the gate time below $500 \mathrm{~ms}$. Figure 13(b) shows the 3rd harmonics of C-mode shows $\sigma_{y}(\tau)<10^{-10}$ for $\tau$ from 20 to $1000 \mathrm{~ms}$, and the fundamental B-mode $\sigma_{y}(\tau)<10^{-10}$ for $\tau$ from 20 to $1000 \mathrm{~ms}$, indicating high stability of the simultaneous multimode oscillation comparable to the single mode oscillation.

\subsection{Temperature Dependence of Oscillation Frequency}

Temperature dependence of the oscillation frequency was measured in the simultaneous oscillation. Allan standard deviation was observed to avoid spontaneous mode change of additional resonance mode. Figure 14 shows root Allan variance at the start point and at higher end of the temperature range. Figure 15 shows the temperature dependence of the oscillation frequency observed simultaneously.

The regression curve of $\mathrm{C}$-mode shows a cubic function with an inflection point at $85.7^{\circ} \mathrm{C}$ (Designed value). The frequency drift of C-mode is $0.32 \times 10^{-6}$ per degree Celsius. This result suggests that the stability of the oscillator remains at the level of typical quartz crystal oscillator. The regression curve of B-mode indicates a linear dependence of approximately $-26.5 \mathrm{ppm} /$ degree. The dual-mode oscillation direct thermometry is applicable over this temperature range.

\subsection{Suppression of the Fundamental B-Mode Oscillations}

Figure 16 shows a typical example of the spectrum of the dual mode quartz crystal oscillation. The spectrum was measured by real time FFT analysis (Rohde-Schwarz RT1004, Rhode and Schwarz Company, Munich, Germany).

Figure 16(a) shows the combination of the 3rd harmonics of C-mode and B-mode, and Figure 16(b) shows the case of the combination of the 3rd harmonics of C-mode and fundamental B-mode. Compared with the separation of frequency between the 3rd harmonics, the separation between the 3rd harmonics oscillation of $\mathrm{C}$-mode and fundamental oscillation of B-mode is $6.2 \mathrm{MHz}$. This wide separation enables easier construction of the mode discrimination system, but this scheme results in more numbers of spectral peaks.

The common part of circuit constants for the analysis are as follows: Oscillator 1: $C_{02}$ and $C_{03}=47 \mathrm{pF} ; C_{0 s}=5$ $\mathrm{pF} ; C_{0 y}=10 \mathrm{pF} ; G_{1 M}=5 \mathrm{~mA} / \mathrm{V}$. Circuit constant of Oscillator 2: $C_{12}$ and $C_{13}=47 \mathrm{pF} ; C_{1 \mathrm{~s}}=5 \mathrm{pF} ; C_{1 y}=10 \mathrm{pF}$; $G_{2 M}=5 \mathrm{~mA} / \mathrm{V} ; C_{z}=100 \mathrm{pF} ; C_{p}=2 \mathrm{pF} ; C_{0 c}=3.431 \mathrm{pF} ; C_{0 b}=3.244 \mathrm{pF} . L_{02}=5.6 \mu \mathrm{H} ; L_{12}=5.6 \mu \mathrm{H} ; C_{0 x}=9 \mathrm{pF} ;$ $C_{1 x}=17 \mathrm{pF} ; L_{02}=33 \mu \mathrm{H}$ for the fundamental mode or $L_{02}=5.6 \mu \mathrm{H}$ for the 3rd harmonic resonance of B-mode.

The common part of circuit constants for this experimental part is as follows. Oscillator 1: $C_{02}$ and $C_{03}=47$ $\mathrm{pF} ; C_{0 y}=10 \mathrm{pF} ; L_{02}=5.6 \mu \mathrm{H} ; C_{0 x}=9 \mathrm{pF}$ for the 3rd harmonic resonance and $L_{02}=33 \mu \mathrm{H} ; C_{0 x}=30 \mathrm{pF}$ for the 


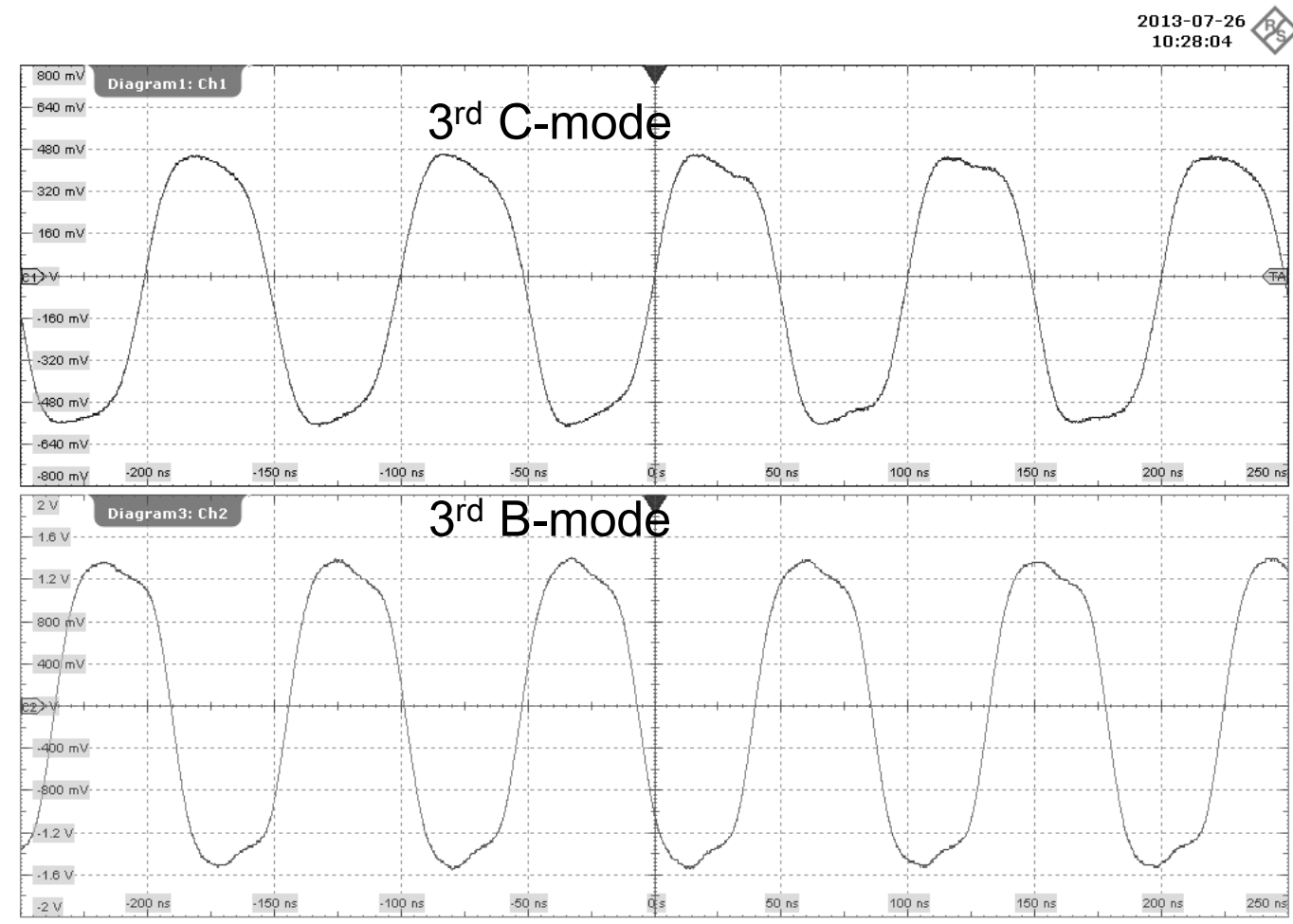

(a)

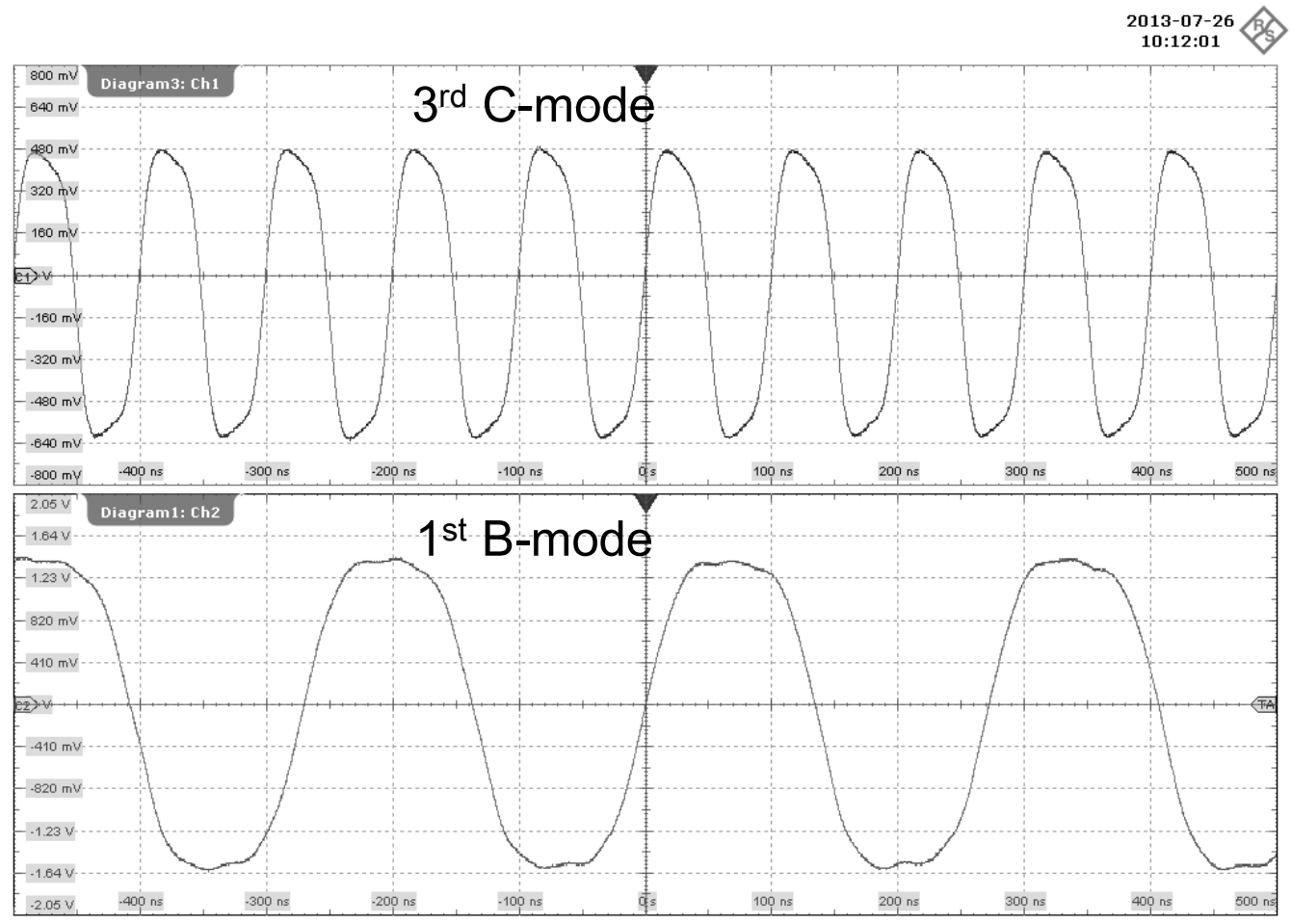

(b)

Figure 11. Observation of simultaneous dual-mode oscillation of SC-cut quartz crystal resonator. (a) Upper track: C-mode 3rd harmonics, $9.999331 \mathrm{MHz}$. Lower track: B-mode 3rd harmonics, 10.885515 MHz. Horizontal scale: $100 \mathrm{~ns} / \mathrm{div}$; (b) Upper track: C-mode, 3rd harmonics, $9.999733 \mathrm{MHz}$, Lower track: B-mode, fundamental, $3.691164 \mathrm{MHz}$. Horizontal scale: 200 ns/div. 


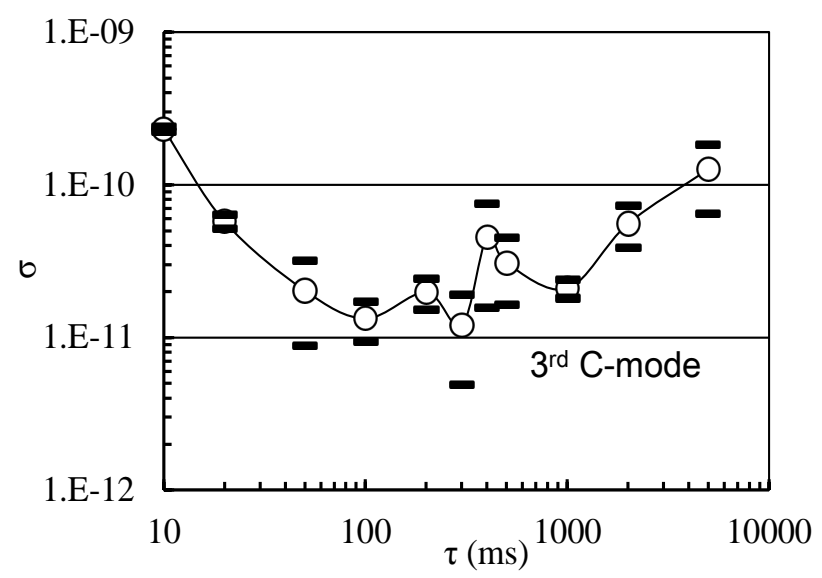

Figure 12. $\sigma$ versus $\tau$ curve of single mode oscillation of Cmode. Open circles: C-mode (9.999835 MHz).

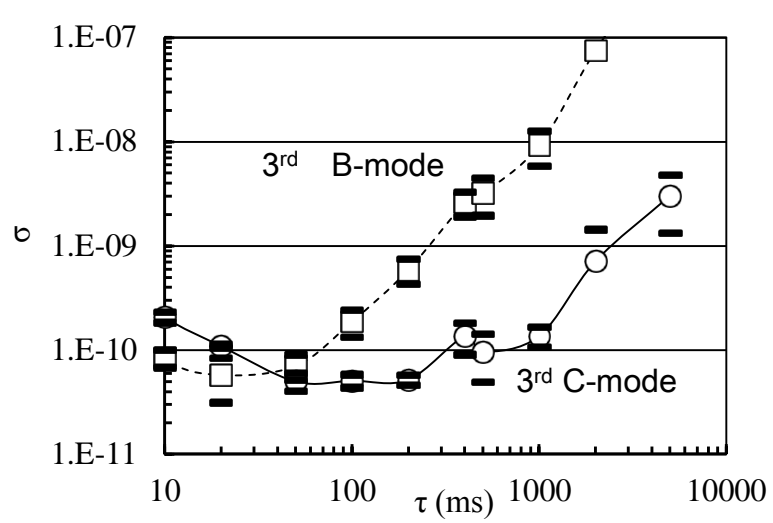

(a)

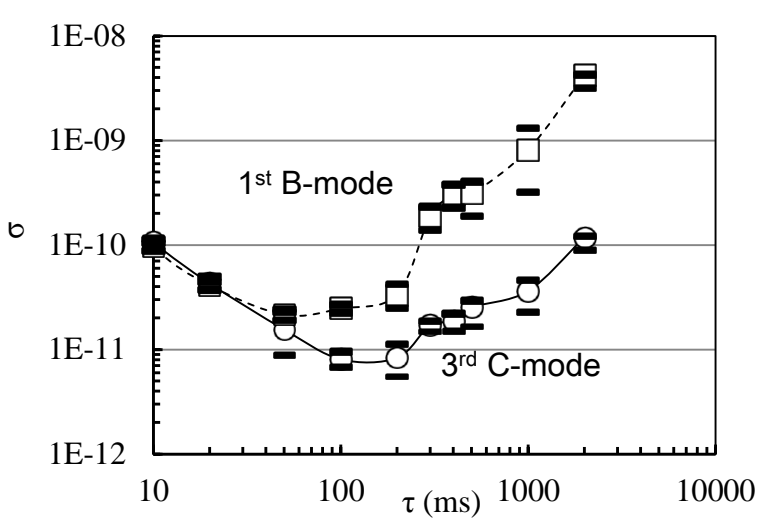

(b)

Figure 13. $\sigma$ versus $\tau$ curve of simultaneous dual-mode oscillation. Open circles: C-mode. Open square symbols: B-mode. Bars: Standard deviation of 5 sequential measurement. (a) $\sigma$ versus $\tau$ curve of C-mode (3rd harmonics, 9.999331 MHz), B-mode (3rd harmonics, $10.885515 \mathrm{MHz}$ ); (b) $\sigma$ versus $\tau$ curve of C-mode at (3rd harmonics, $9.999733 \mathrm{MHz}$ ), B-mode (fundamental, $3.691164 \mathrm{MHz})$.

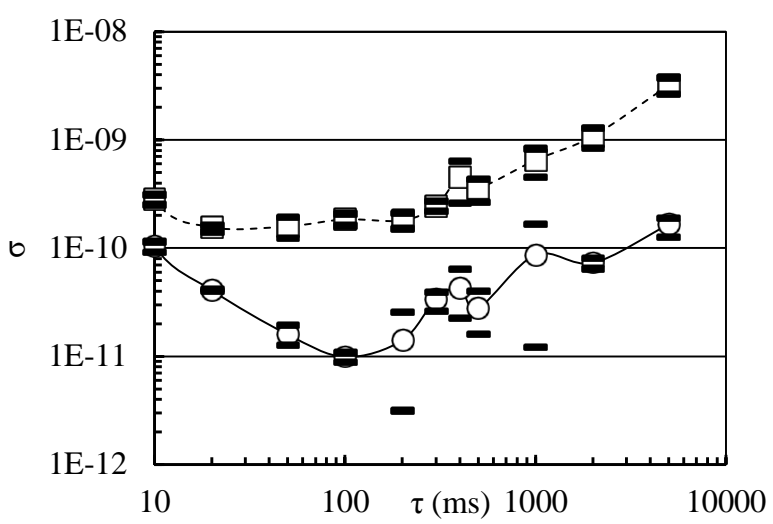

(a)

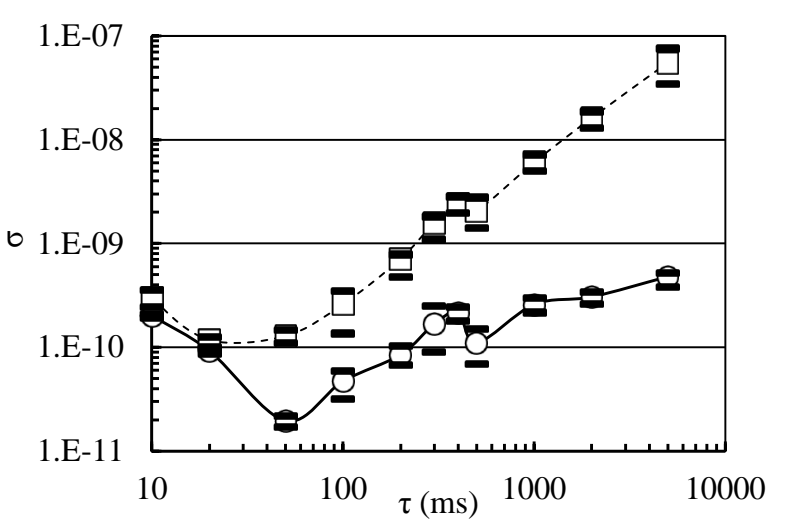

(b)

Figure 14. $\sigma$ versus $\tau$ curve at the start and the endpoint of the temperature dependence. Open circles: C-mode. Open square symboles: B-mode. Bar: Standard deviation of 5 sequential data. (a) $\sigma$ versus $\tau$ curve of C-mode (3rd harmonics, 9.999331 $\mathrm{MHz}$ ), B-mode (3rd harmonics, $10.885515 \mathrm{MHz}$ ) at $27^{\circ} \mathrm{C}$; (b) $\sigma$ versus $\tau$ curve of C-mode (3rd harmonics, $9.9999956 \mathrm{MHz}$ ), B-mode (3rd harmonics, $10.963845 \mathrm{MHz}$ ) at $80^{\circ} \mathrm{C}$. 


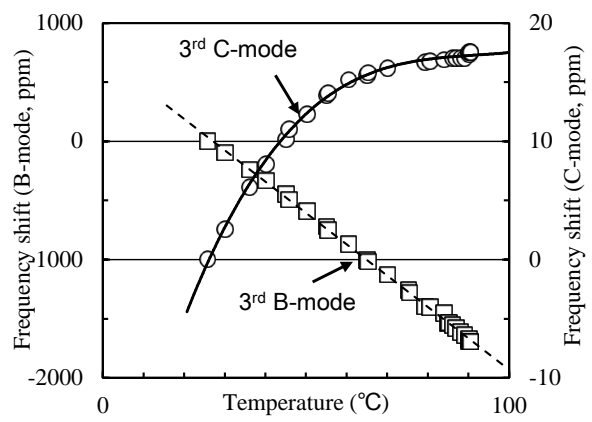

Figure 15. Simultaneously measured oscillation frequency. Open circles: C-mode. Open square symbols: B-mode. Regression function of B-mode: $\mathrm{Y}=-26.453 \mathrm{X}+716.55$.

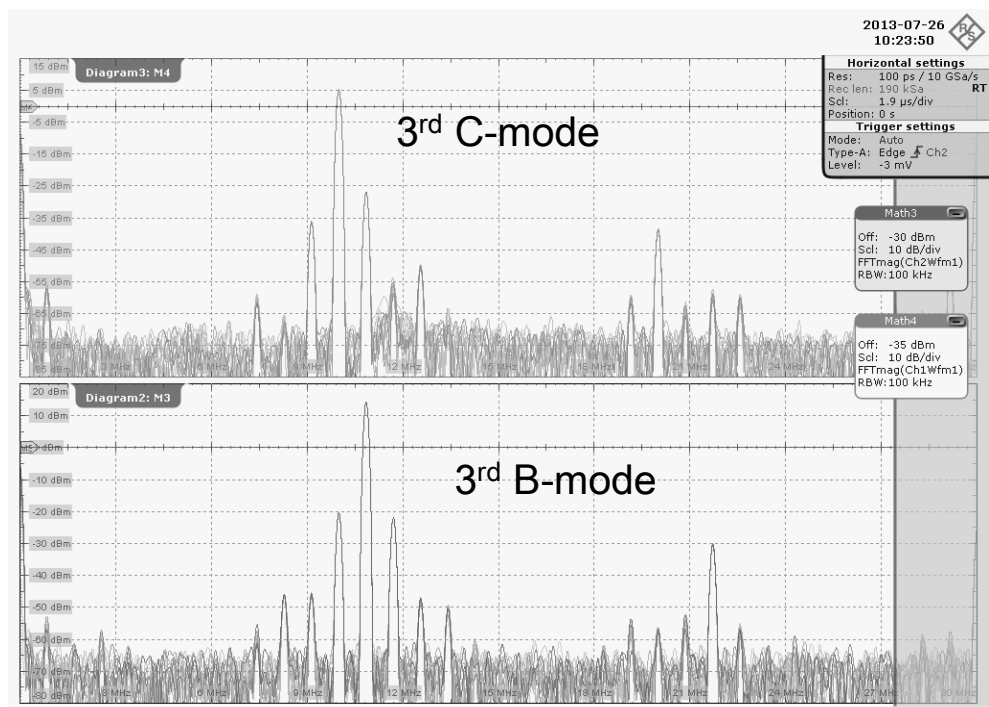

(a)

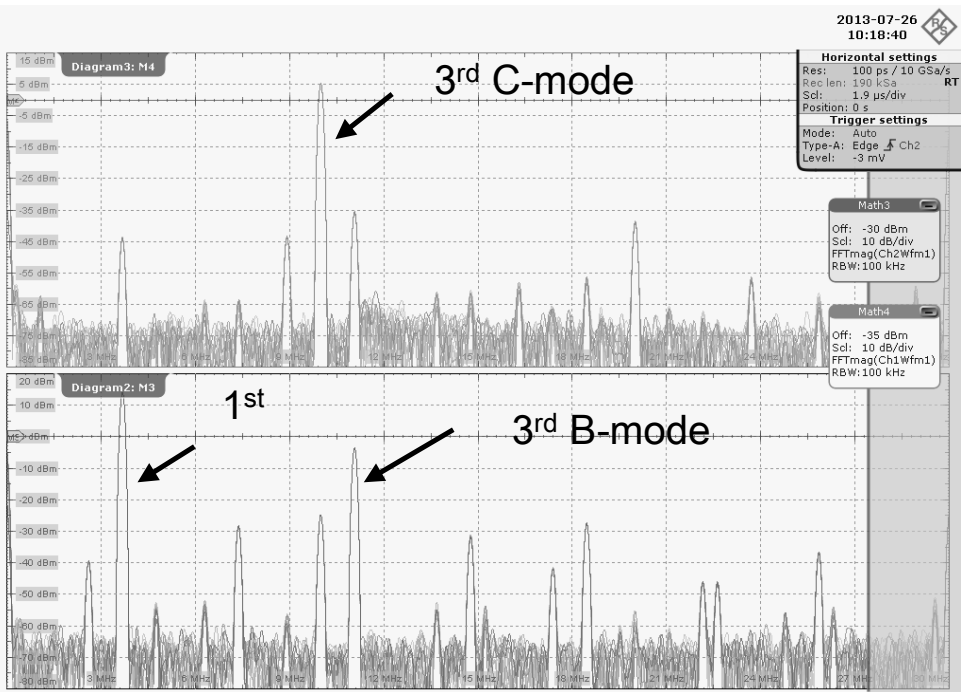

(b)

Figure 16. Spectrum of the simultaneous oscillation. Horizontal scale: Span 0 to $30 \mathrm{MHz}$. Resolution $100 \mathrm{kHz}$. (a) Spectrum of C-mode (3rd harmonics, 9.99975 MHz upper track) and B-mode (3rd harmonics, $10.97758 \mathrm{MHz}$ lower track); (b) Spectrum of C-mode (3rd harmonics, 9.99975 MHz, upper track) and B-mode (fundamental, 3.69147 MHz, lower track). 
fundamental resonance of B-mode. Oscillator 2: $C_{12}$ and $C_{13}=47 \mathrm{pF} ; C_{1 y}=10 \mathrm{pF} ; L_{12}=5.6 \mu \mathrm{H} ; C_{1 x}=17 \mathrm{pF}$ for the 3rd harmonic resonance of C-mode. $\mathrm{IC}_{1}$ and $\mathrm{IC}_{2} \mathrm{TC} 7 \mathrm{SHU} 04 \mathrm{~F}, V_{c c}=5 \mathrm{~V}$.

\section{Conclusions}

The SC-cut resonator is made of double-rotated cut crystals and it shows a principal resonance C-mode and B-mode, additional resonance mode. High stability of the principal mode was observed in both single and multimode resonance oscillation. The linear dependence of the B-mode on the temperature can realized the thermometric method with the assurances of the stability. Design rules were ensured by the measurement of the stability of $\sigma$ versus $\tau$ curve.

The narrow-band wide variable crystal oscillator circuit was proved to be an efficient experimental maneuver in the simultaneous measurement of the stability and the oscillation frequency dependence on the ambient temperature. The frequency separation is narrower than the combination of the 3rd harmonic resonance of $\mathrm{C}$-mode and the fundamental resonance of B-mode which is approximately 6.2 MHz. The combination of the 3rd harmonic resonance oscillation shows quasi-sinusoidal wave forms with negligible distortion with Allan standard deviation $\sigma_{y}(\tau), 10^{-11}<\sigma_{y}(\tau)<10^{-10}$, sufficiently stable for standard time base and sensor applications. In the present stage of the experiment, the demonstrated result was obtained by precise choice of the circuit constant and bias current. It is necessary to monitor the unlocking from crystal resonance by the real-time monitoring of the Allan standard deviation. Although the reproducibility of the circuit and the oscillation condition is not thoroughly identified and appropriate circuit design is still necessary, we risk concluding that the dual-mode quartz crystal resonance oscillation can be applied to piezoelectric sensing and the production of a stable frequency standard.

\section{Acknowledgements}

We express acknowledgement to Ms Ruzaini Izyan binti Ruslan and Ms Izzati Syazwani binti Othman for their collaboration in the early stage of experiment, and Mr. Masahide Marumo, River Eletec Company (Japan) for fruitful discussions.

\section{References}

[1] Vig, J.R. (1999) Dual-Mode Oscillators for Clocks and Sensors. Proceedings of the 1999 IEEE Ultrasonics Symposium, Caesars Tahoe, 17-20 October 1999, 859-868. http://dx.doi.org/10.1109/ULTSYM.1999.849128

[2] Vig, J.R. (2001) Temperature Insensitive Dual-Mode-Resonant Sensors-A Review. IEEE Sensors Journal, 1, $62-68$. http://dx.doi.org/10.1109/JSEN.2001.923588

[3] Schodowski, S.S. (1989) Resonator Self-Temperature Sensing Using a Dual-Harmonic-Mode Oscillator. Proceedings of the 43rd Annual Symposium on Frequency Control, Denver, 31 May-2 June 1989, 2-7. http://dx.doi.org/10.1109/FREQ.1989.68851

[4] Vig, J.R. (2012) Thermometric Beat Frequency Generation. In: Quartz Crystal Resonators and Oscillators for Frequency Control and Timing Applications. A Tutorial, US Army, Communications Electronics Research Development \& Engineering Center (CERDEC), Fort Monmouth, 65-71. http://www.ieee-uffc.org/frequency_control/teaching/vig/vig3.ppt-2012-05-14

[5] Štofanik, V., Baláž, I., Minárik, M. and Cocherová, E. (2008) Investigation of Self-Temperature-Sensing of SC-Cut with Simultaneous Excitation of Two Higher Overtones in Dual-Mode Crystal Oscillator. IEEE International Frequency Control Symposium, Honolulu, 19-21 May 2008, 170-173.

[6] Ferrari, M., Ferrari, V. and Kanazawa, K.K. (2008) Dual Harmonic Oscillator for Quartz Crystal Resonator Sensors. Sensors and Actuators A, 145-146, 131-138.

[7] Izyan-Ruslan, R., Sato, T. and Akitsu, T. (2012) Voltage Controlled Narrow Band Wide-Variable Range Four Segment Quartz Crystal Oscillator. Transactions Ultrasonics, Ferro-Electrics and Frequency Control, 59, 564-572.

[8] Barnes, J.A., Chi, A.R., Cutler, L.S., Healey, D.J., Leeson, D.B., McGunigal, T.E., Mullen Jr., J.A., Smith, W.L., Sydnor, R.L., Vessot, R.F.C. and Winkler, G.M.R. (1971) Characterization of Frequency Stability. IEEE Transactions on Instrumentation and Measurement, IM-20, 105-120.

[9] Allan, D.W. (1966) Statistics of Atomic Frequency Standards. Proceedings of the IEEE, 54, 221-230. http://dx.doi.org/10.1109/PROC.1966.4634

[10] 1139-1999 IEEE Standard Definitions of Physical Quantities for Fundamental Frequency and Time Metrology. 
Scientific Research Publishing (SCIRP) is one of the largest Open Access journal publishers. It is currently publishing more than 200 open access, online, peer-reviewed journals covering a wide range of academic disciplines. SCIRP serves the worldwide academic communities and contributes to the progress and application of science with its publication.

Other selected journals from SCIRP are listed as below. Submit your manuscript to us via either submit@scirp.org or Online Submission Portal.
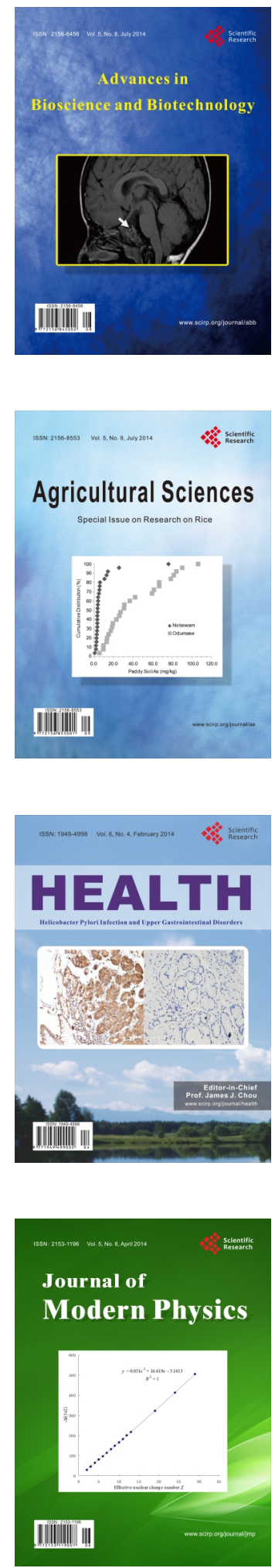
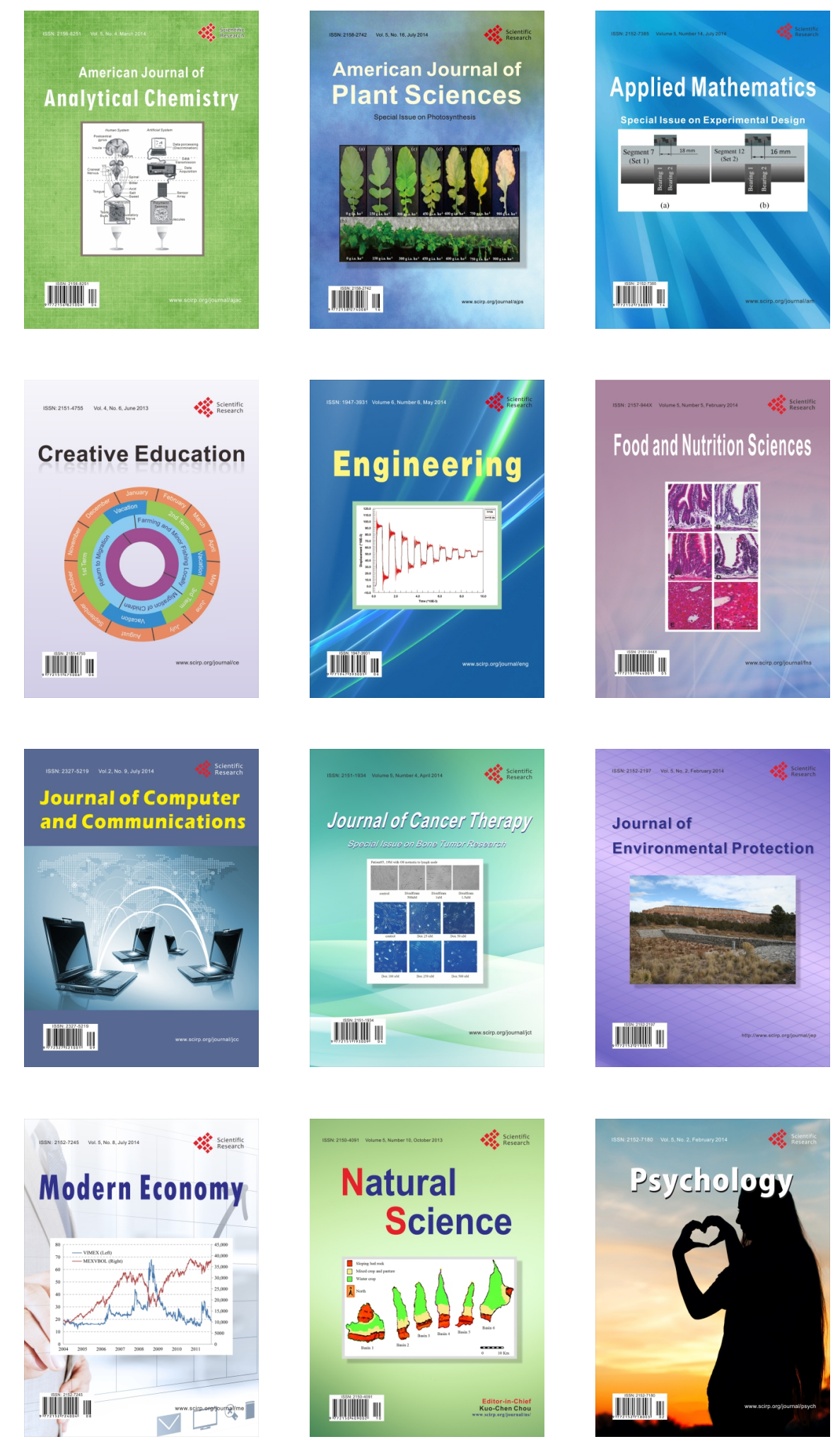